\title{
Prostate cancer incidence in men with self-reported prostatitis after 15 years of follow-up
}

\author{
MARKKU H. VAARALA, AARE MEHIK, PASI OHTONEN and PEKKA A. HELLSTRÖM
}

Department of Surgery, Medical Research Center Oulu, Oulu University Hospital, University of Oulu, 90029 Oulu, Finland

Received May 19, 2015; Accepted April 15, 2016

DOI: $10.3892 / 01.2016 .4702$

\begin{abstract}
Controversy exists regarding a possible association between prostatitis and prostate cancer. To further evaluate the incidence of prostate cancer following prostatitis, a study of prostate cancer incidence in a cohort of Finnish men was performed. The original survey evaluating self-reported prostatitis was conducted in 1996-1997. A database review was conducted focusing on prostate cancer diagnoses in the cohort. In 2012, there were $13(5.2 \%)$ and $27(1.8 \%)$ prostate cancer cases among men with $(n=251)$ and without $(n=1,521)$ prostatitis symptoms, respectively. There were no significant differences in age, primary therapy distribution, prostate-specific antigen levels, Gleason score, clinical T-class at the time of prostate cancer diagnosis, or time lag between the original survey and prostate cancer diagnosis. The standardized incidence ratio (SIR) of prostate cancer was 1.16 [95\% confidence interval (CI), 0.62-1.99] and 0.44 (95\% CI, 0.29- 0.64) among men with and without prostatitis symptoms, respectively. After 15 years of follow-up subsequent to self-reported prostatitis, no evident increase in incidence of prostate cancer was detected among Finnish men with prostatitis symptoms. The higher percentage of prostate cancer among men with prostatitis symptoms appears to be due to coincidentally low SIR of prostate cancer among men without prostatitis symptoms, and may additionally be due to increased diagnostic examinations. Further research is required to confirm this speculation.
\end{abstract}

\section{Introduction}

Chronic inflammation is associated with the development of multiple types of cancer, but there are discrepancies between different studies concerning prostate cancer (1). Two previous meta-analyses provided significant evidence of the association between prostatitis and prostate cancer $(1,2)$. There are, however, several studies showing no association or a negative

Correspondence to: Dr Markku H. Vaarala, Department of Surgery, Medical Research Center Oulu, Oulu University Hospital, University of Oulu, Kajaanintie 50, 90029 Oulu, Finland

E-mail:markku.vaarala@oulu.fi

Key words: prostatitis, prostate cancer, inflammation, epidemiology correlation between prostatitis and prostate cancer (3-7). It has been proposed that chronic inflammatory infiltrate-positive prostatitis may protect against prostate cancer (8). The majority of previous studies included patients with diagnosis of prostate cancer and controls from patients records or interviews $(1,2,9)$. By contrast, the present study used a randomly selected sample of Finnish men surveyed in the 1990s to evaluate the effect of prostatitis on prostate cancer incidence.

\section{Materials and methods}

Patients. From April 1996 to February 1997, a survey was conducted in the northern region of Finland to evaluate the prevalence of prostatitis. A total of 2,500 men aged 20-59 years were randomly selected from the population registry (Population Register Centre, Helsinki, Finland) to receive a questionnaire, and 1,832 of them responded. A database review was then performed focusing on prostate cancer diagnoses in the cohort. Respondents were identified via a personal identification number from the Oulu University Hospital registry (Oulu, Finland), based on the name and address information available at the time of the original survey. When present, prostate cancer diagnoses and diagnosis years for the surveyed men were obtained from the Finnish Cancer Registry (Helsinki, Finland). Clinical characteristics [Gleason score, T-class, prostate-specific antigen (PSA) levels and primary therapy] of prostate cancer cases were reviewed from the patients' medical charts.

Ethics statement. The local ethics council of Oulu University Hospital (Oulu, Finland) approved the present study, which was conducted according to the Declaration of Helsinki (10). Written consent from the patients was not obtained, but the volunteers responding to the original survey (11) was considered as consent to participate in the present study. The National Institute for Health and Welfare (Helsinki, Finland), approved the present study and the use of registry data following local ethical approval, according to the Finnish law. Patient data was anonymized and de-identified prior to statistical analysis.

Statistics. Summary statistics included the mean and standard deviation (SD), or the median with the 25th-75th percentile if biased, unless otherwise stated. Comparisons for categorical data were performed using the $\chi^{2}$ test or the Fisher's exact test. Continuous variables were analyzed 
using the Mann-Whitney U non-parametric test. Prostate cancer incidence and standardized incidence ratio (SIR) with 95\% confidence interval (CI) were calculated for the study population and for the whole population of the survey area, which included men aged 41-80 years living at the study area (obtained from the Statistics Finland database) (12) and the number of newly diagnosed prostate cancer cases (obtained from the Finnish Cancer Registry). Data were analyzed using SPSS statistical software version 22.0 (IBM SPSS, Armonk, NY, USA). Two-tailed $\mathrm{P}$-values are reported and $\mathrm{P}<0.05$ were considered to indicate a statistically significant difference.

\section{Results}

Of the 1,832 men responding to the original survey, 261 had prostatitis symptoms, leading to a lifetime prevalence of $14.2 \%$ (13). In the present study, detailed data were available for 251 and 1,772 out of 261 and 1,832 men with prostatitis symptoms and the total number of men responding to the original survey, respectively. Missing cases $(n=60)$ were due to incomplete identification data recorded following the original survey. According to the Finnish Cancer Registry, there were a total of 40 prostate cancer cases diagnosed among men in the cohort in 2012. The incidence of prostate cancer was more than double among men reporting prostatitis symptoms in the original survey (Table I).

There was no significant difference in the ages of men at the time of prostate cancer diagnosis between the groups. Mean ages (SD, range) were 64.8 years $(3.7,58-73$ years) and 62.7 years $(6.2,51-73$ years) for subjects with and without prostatitis symptoms, respectively $(\mathrm{P}=0.26)$. PSA values at prostate cancer diagnosis were available for 13 and 24 subjects with and without prostatitis symptoms, respectively. Median PSA values (25th-75th percentile, range) were $8.1 \mu \mathrm{g} / 1$ (5.2-13.2, $0.4-26.0 \mu \mathrm{g} / \mathrm{l})$ and $9.2 \mu \mathrm{g} / 1(5.9-18.9,0.9-1,759.0 \mu \mathrm{g} / \mathrm{l})$ for subjects with and without prostatitis symptoms, respectively $(\mathrm{P}=0.33)$. The Gleason score at diagnosis was available for 13 and 25 subjects with and without prostatitis symptoms, respectively. The median Gleason scores (25th-75th percentile, range) were $6(6-7,4-8)$ and $6(6-8,4-9)$ for subjects with and without prostatitis symptoms, respectively $(\mathrm{P}=0.55)$.

Table II contains the distribution of clinical T-class (tumor-node-metastasis classification) (14) among men with prostate cancer. Although there was a tendency for an increased number of subjects with locally advanced disease (T3-T4) among men with prostatitis symptoms, the difference was not significant $(\mathrm{P}=0.63)$. There were no data available for one subject. The distribution of different primary treatment modalities did not differ between the groups $(\mathrm{P}=0.61)$. There were no data available for one subject. Furthermore, there was no significant difference between the groups in the time lag between the survey and the diagnosis of prostate cancer $(\mathrm{P}=0.79)$.

The present study further evaluated the incidence of prostate cancer in the present cohort compared with that observed during 15 years in the geographical area where the original survey was conducted. Despite the seemingly high incidence of prostate cancer among men with prostatitis symptoms, the incidence was not higher than that reported among men in the age groups of 61-70 and 71-80 years in the aforementioned
Table I. Incidence of prostate cancer among Finnish men with and without self-reported prostatitis symptoms.

\begin{tabular}{lccr}
\hline & \multicolumn{2}{c}{ Prostatitis symptoms } & \\
\cline { 2 - 3 } Patients & No,n $(\%)$ & Yes, n (\%) & Total, n \\
\hline $\begin{array}{l}\text { Prostate cancer } \\
\text { diagnosis }\end{array}$ & & \\
No & $1,494(98.2)$ & $238(94.8)$ & 1,732 \\
Yes & $27(1.8)$ & $13(5.2)^{\mathrm{a}}$ & 40 \\
Total & 1,521 & 251 & 1,772 \\
\hline
\end{tabular}

${ }^{\mathrm{a}} \mathrm{P}=0.001$.

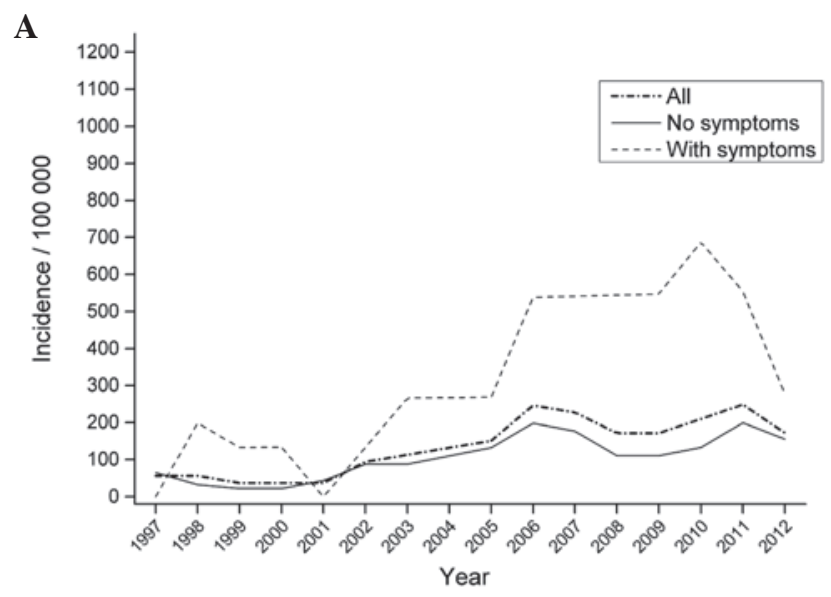

B

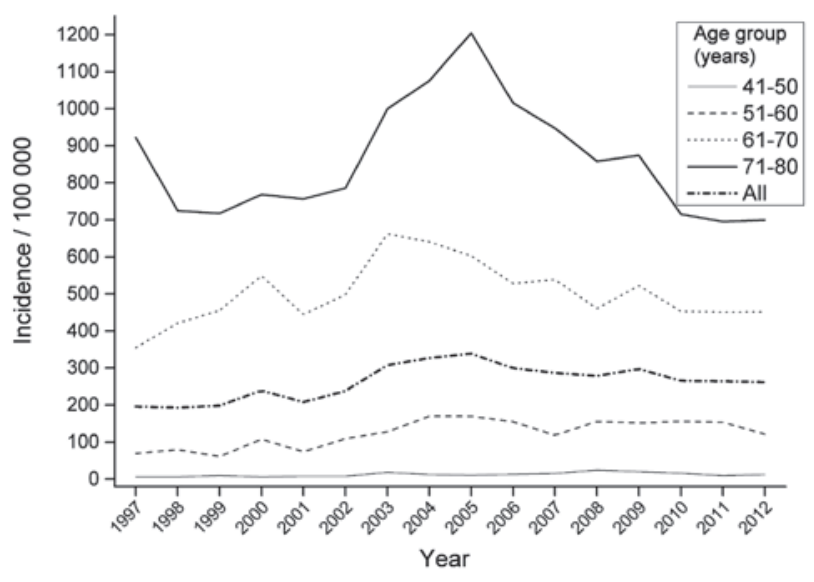

Figure 1. (A) Incidence of prostate cancer among the study cohort. Annual incidence of prostate cancer (number of cases $/ 100,000$ ) among a cohort of Finnish men $(n=1,772)$ with or without self-reported symptoms of prostatitis. The time series was smoothed by calculating the 3-year moving average for each year. (B) Annual incidence of prostate cancer in the geographical area of the original survey. Curves for different age groups and the combined curve for all presented age groups are shown.

geographical area (Fig. 1). Furthermore, the analysis of SIR of prostate cancer revealed that the SIR was slightly increased among men with prostatitis symptoms, but the $95 \% \mathrm{CI}$ covered 1.0, indicating no significant difference compared with the population. The SIR of prostate cancer among men with no prostatitis symptoms was lower than expected (Table III). 
Table II. Clinical T-class, primary prostate cancer therapy and year of prostate cancer diagnosis among Finnish men with prostate cancer with or without a history of self-reported prostatitis.

\begin{tabular}{|c|c|c|c|}
\hline \multirow[b]{2}{*}{ Characteristics } & \multicolumn{2}{|c|}{ Prostatitis symptoms } & \multirow[b]{2}{*}{ Total } \\
\hline & No, n $(\%)$ & Yes, n $(\%)$ & \\
\hline \multicolumn{4}{|l|}{ Clinical T-class } \\
\hline T1a-T1c & $6(22.2)$ & $3(25.0)$ & 9 \\
\hline $\mathrm{T} 2$ & $10(37.0)$ & $6(50.0)$ & 16 \\
\hline T3-T4 & $11(40.7)$ & $3(25.0)$ & 14 \\
\hline \multicolumn{4}{|l|}{ Primary prostate cancer therapy } \\
\hline Active surveillance & $1(3.7)$ & $2(16.7)$ & 3 \\
\hline Permanent seed implantation radiation therapy & $5(18.5)$ & $1(8.3)$ & 6 \\
\hline Androgen deprivation therapy & $5(18.5)$ & $3(25.0)$ & 8 \\
\hline Radical prostatectomy & $6(22.2)$ & $2(16.7)$ & 8 \\
\hline External beam radiation therapy & $10(37.0)$ & $4(33.3)$ & 14 \\
\hline \multicolumn{4}{|l|}{ Year of prostate cancer diagnosis } \\
\hline 1997 & $1(3.7)$ & $0(0.0)$ & 1 \\
\hline 1998 & $0(0.0)$ & $1(7.7)$ & 1 \\
\hline 2000 & $1(3.7)$ & $0(0.0)$ & 1 \\
\hline 2001 & $1(3.7)$ & $0(0.0)$ & 1 \\
\hline 2002 & $2(7.4)$ & $1(7.7)$ & 3 \\
\hline 2003 & $1(3.7)$ & $1(7.7)$ & 2 \\
\hline 2004 & $2(7.4)$ & $0(0.0)$ & 2 \\
\hline 2005 & $3(11.1)$ & $1(7.7)$ & 4 \\
\hline 2006 & $4(14.8)$ & $3(23.1)$ & 7 \\
\hline 2007 & $1(3.7)$ & $0(0.0)$ & 1 \\
\hline 2008 & $0(0.0)$ & $1(7.7)$ & 1 \\
\hline 2009 & $4(14.8)$ & $3(23.1)$ & 7 \\
\hline 2010 & $2(7.4)$ & $1(7.7)$ & 3 \\
\hline 2011 & $3(11.1)$ & $0(0.0)$ & 3 \\
\hline 2012 & $2(7.4)$ & $1(7.7)$ & 3 \\
\hline
\end{tabular}

Table III. SIRs of men with and without prostatitis symptoms in different age groups in the geographical area of the study.

\begin{tabular}{|c|c|c|c|c|c|c|c|c|}
\hline \multirow[b]{3}{*}{$\begin{array}{l}\text { Age group, } \\
\text { years }\end{array}$} & \multirow{2}{*}{\multicolumn{2}{|c|}{$\begin{array}{l}\text { Prostate cancer cases in } \\
\text { the study population, } n\end{array}$}} & \multirow{2}{*}{\multicolumn{2}{|c|}{$\begin{array}{l}\text { Expected cases in } \\
\text { the study population, } \mathrm{n}\end{array}$}} & \multicolumn{4}{|c|}{ SIRs } \\
\hline & & & & & \multicolumn{2}{|c|}{ Prostatitis symptoms } & \multicolumn{2}{|c|}{ No symptoms } \\
\hline & $\begin{array}{l}\text { Prostatitis } \\
\text { symptoms }\end{array}$ & $\begin{array}{c}\text { No } \\
\text { symptoms }\end{array}$ & $\begin{array}{l}\text { Prostatitis } \\
\text { symptoms }\end{array}$ & $\begin{array}{c}\text { No } \\
\text { symptoms }\end{array}$ & $1 / 100,000$ & $95 \% \mathrm{CI}$ & $1 / 100,000$ & $95 \% \mathrm{CI}$ \\
\hline $41-50$ & 0 & 0 & 0.08 & 1.03 & 0.00 & $0.00-46.40$ & 0.00 & $0.00-3.57$ \\
\hline $51-60$ & 1 & 10 & 1.84 & 14.20 & 0.55 & $0.01-3.04$ & 0.70 & $0.34-1.29$ \\
\hline $61-70$ & 11 & 13 & 6.87 & 35.30 & 1.60 & $0.80-2.87$ & 0.37 & $0.20-0.63$ \\
\hline $71-80$ & 1 & 4 & 2.40 & 11.30 & 0.42 & $0.01-2.32$ & 0.35 & $0.10-0.91$ \\
\hline Total, n & 13 & 27 & 11.20 & 61.90 & 1.16 & $0.62-1.99$ & 0.44 & $0.29-0.64$ \\
\hline
\end{tabular}

SIR, standardized incidence ratio.

\section{Discussion}

To the best of our knowledge, the present study is the first to evaluate a large cohort of randomly selected men for several years following the report of prostatitis symptoms in order to measure the risk of developing prostate cancer. The prevalence of self-reported prostatitis in the present cohort was $14.2 \%$ (11), which is consistent with a previous survey conducted by health 
professionals in the USA, where the prevalence of prostatitis was $16 \%$ (13), thus supporting the validity of the present cohort. Previously, an association between self-reported prostatitis and self-reported prostate cancer was documented (15); however, that study was not longitudinal, in contrast to the current study. Additionally, a previous retrospective study among men with prostate cancer revealed an elevated incidence of history of any type of prostatitis compared with matched control men (9). On the contrary, histological prostatitis has been reported to be significantly more prevalent in benign prostatic hyperplasia than in prostate cancer (16). However, it is well known that histological prostatitis does not correlate with clinical symptoms (17).

In the present study, the time lag between the original survey and the diagnosis of prostate cancer was long (Table I), which further challenges the connection between prostatitis symptoms and prostate cancer. However, in another study, the mean time from the most recent episode of acute prostatitis and the diagnosis of prostate cancer was 12.2 years among a cohort of prostate cancer patients (9). Based on the present data, it is possible to suggest that the increased incidence of prostate cancer among men with prostatitis symptoms compared with that among men with no symptoms may be due to a larger number of prostate cancer diagnostic examinations based on the patients symptoms. Data supporting this hypothesis have been published recently (18). In that study, the increased lower urinary tract symptoms were not associated with the intensity of prostate cancer diagnosis, but the diagnostic intensity increased when symptoms were brought to the attention of physicians (18). However, the present data do not enable the reliable evaluation of this aspect. The current results demonstrated that prostatitis symptoms did not lead to a higher incidence of prostate cancer in the geographical area evaluated after 15 years of follow-up, compared with that in the general population. Furthermore, despite the seemingly higher prostate cancer incidence among men with prostatitis symptoms, the $95 \%$ CIs of SIRs revealed that the differences were not significant, which may be due to the low amount of prostate cancer cases and the limited number of men with prostatitis symptoms in the present study. The current cohort was obtained by random sampling from a population registry. Therefore, the low SIR of prostate cancer among men with no symptoms is likely to be coincidental.

Although there was a significant difference in the number of cancer cases between men with and without a history of prostatitis, the limited number of cancer cases included in the present study prevents any firm conclusions. In the present cohort, a remarkable amount of men were young at the time of the original survey, and were not in the highest risk group for prostate cancer, as estimated by age 15 years later, despite the fact that $\sim 1 / 3$ of the men were 50-59 years old at the time of the original survey (11).

There are several limitations in the present study. Firstly, there was no differentiation between the various types of prostatitis. Therefore, it could not be concluded whether the risk of cancer is different in patients with chronic prostatitis compared with that in men with 1-2 acute episodes of prostatitis. Secondly, the diagnosis of prostatitis was based on a questionnaire, and it has been reported that self-reported genitourinary diseases such as benign prostatic hyperplasia and prostatitis are poorly concordant with data from medical records (19). However, the respondents provided the details of the health care professional (general practitioner or hospital doctor/urologist) who established the diagnosis of prostatitis; thus, the diagnosis was not based solely on patient self-evaluation (11). Possible symptoms at the time of prostate cancer diagnosis were not collected from the patients charts, as the retrospective evaluation of symptoms is likely to be misleading, due to the lack of systematic recording of the presence or absence of symptoms.

The incidence of prostate cancer in the present study was based on data from the Finnish Cancer Registry, which automatically receives notification of each suspected or diagnosed cancer directly from every pathology laboratory (20). However, despite the estimated high consistency of the Finnish Cancer Registry (diagnosed and registered prostate cancer cases, 99\%), a number of prostate cancer diagnoses may be missed (21).

It is challenging to draw conclusive deductions regarding the connection between prostatitis and prostate cancer. Chronic prostatitis is a symptom with no objective diagnostic test. In certain men, chronic pelvic pain may mimic prostatitis with no inflammation of the prostate (22). Thus, including these men will produce bias in similar studies.

To conclude, after 15 years of follow-up subsequent to self-reported prostatitis, no evidently increased incidence of prostate cancer was detected in the present cohort of Finnish men. Despite the higher percentage of prostate cancer among men with prostatitis symptoms compared with that among men with no symptoms, the SIR of prostate cancer among men with prostatitis symptoms was within the expected range of values. It may be suggested that the higher percentage of prostate cancer among men with prostatitis symptoms compared with that among men without symptoms is due to the low SIR of prostate cancer cases among men without prostatitis symptoms, and it may also be due to more frequent prostate cancer diagnostic examinations based on symptoms. The present results do not support extensive diagnostic interventions in order to detect possible prostate cancer among men with prostatitis symptoms, considering that the clinical characteristics of prostate cancer did not differ between men with and without prostatitis symptoms.

\section{Acknowledgements}

The present authors would like to thank Mrs. Leena Heikkilä (Oulu University Hospital, Oulu, Finland) for her technical assistance and Dr Maarit Leinonen (Finnish Cancer Registry, Helsinki, Finland) for her assistance with the Finnish Cancer Registry data.

\section{References}

1. Dennis LK, Lynch CF and Torner JC: Epidemiologic association between prostatitis and prostate cancer. Urology 60: 78-83, 2002.

2. Jiang J, Li J, Yunxia Z, Zhu H, Liu J and Pumill C: The role of prostatitis in prostate cancer: Meta-analysis. PLoS One 8: e85179, 2013

3. Gerstenbluth RE, Seftel AD, MacLennan GT, Rao RN, Corty EW Ferguson K and Resnick MI: Distribution of chronic prostatitis in radical prostatectomy specimens with up-regulation of bcl-2 in areas of inflammation. J Urol 167: 2267-2270, 2002.

4. Irani J, Goujon JM, Ragni E,Peyrat L, Hubert J, Saint F and Mottet N; Pathologist Multi Center Study Group: High-grade inflammation in prostate cancer as a prognostic factor for biochemical recurrence after radical prostatectomy. Urology 54: 467-472, 1999. 
5. Engelhardt PF, Brustmann H, Seklehner S and Riedl CR: Chronic asymptomatic inflammation of the prostate type IV and carcinoma of the prostate: Is there a correlation? Scand J Urol 47: 230-235, 2013.

6. Karakiewicz PI, Benayoun S, Bégin LR, Duclos A, Valiquette L, McCormack M, Bénard F, Saad F and Perrotte P: Chronic inflammation is negatively associated with prostate cancer and high-grade prostatic intraepithelial neoplasia on needle biopsy. Int J Clin Pract 61: 425-430, 2007.

7. Moreira DM, Nickel JC, Gerber L, Muller RL, Andriole GL, Castro-Santamaria R and Freedland SJ: Baseline prostate inflammation is associated with a reduced risk of prostate cancer in men undergoing repeat prostate biopsy: Results from the REDUCE study. Cancer 120: 190-196, 2014.

8. Porcaro AB, Rubilotta E, Petrozziello A, Ghimenton C, Migliorini F, Zecchini Antoniolli S, Lacola V, Monaco C, Curti P, Cavalleri S, et al: Chronic inflammation of the prostate type IV with respect to risk of prostate cancer. Arch Ital Urol Androl 86: 208-211, 2014

9. Roberts RO, Bergstralh EJ, Bass SE, Lieber MM and Jacobsen SJ: Prostatitis as a risk factor for prostate cancer. Epidemiology 15 93-99, 2004.

10. WMA Declaration of Helsinki - Ethical Principles for Medical Research Involving Human Subjects. www.wma. net/en/30publications/10policies/b3/. Accessed Apr 15, 2016.

11. Mehik A, Hellström P, Lukkarinen O, Sarpola A and Järvelin M: Epidemiology of prostatitis in Finnish men: A population-based cross-sectional study. BJU Int 86: 443-448, 2000.

12. Statistics Finland. www.tilastokeskus.fi/index en.html. Accessed Apr 15, 2016

13. Collins MM, Meigs JB, Barry MJ, Walker Corkery E, Giovannucci E and Kawachi I: Prevalence and correlates of prostatitis in the health professionals follow-up study cohort. J Urol 167: 1363-1366, 2002
14. Sobin L and Wittekind C: Union for International Cancer Control: TNM Classification of Malignant Tumours. 6th edition. Wiley, New York, 2002.

15. Daniels NA, Ewing SK, Zmuda JM, Wilt TJ and Bauer DC; Osteoporotic Fractures in Men (MrOS) Research Group: Correlates and prevalence of prostatitis in a large community-based cohort of older men. Urology 66: 964-970, 2005.

16. Edlin RS, Heyns CF, Van Vuuren SP and Zarrabi AD: Prevalence of histological prostatitis in men with benign prostatic hyperplasia or adenocarcinoma of the prostate presenting without urinary retention. S Afr J Surg 50: 127-130, 2012.

17. Nickel JC, Roehrborn CG, O'leary MP, Bostwick DG, Somerville MC and Rittmaster RS: Examination of the relationship between symptoms of prostatitis and histological inflammation: Baseline data from the REDUCE chemoprevention trial. J Urol 178: 896-901, 2007.

18. Weight CJ, Kim SP, Jacobson DJ, McGree ME, Boorjian SA, Thompson RH, Leibovich BC, Karnes RJ and St Sauver J: The effect of benign lower urinary tract symptoms on subsequent prostate cancer testing and diagnosis. Eur Urol 63: 1021-1027, 2013.

19. Zhu K, McKnight B, Stergachis A, Daling JR and Levine RS: Comparison of self-report data and medical records data: Results from a case-control study on prostate cancer. Int J Epidemiol 28: 409-417, 1999.

20. Finnish Cancer Registry - Legislation. www.cancer.fi/syoparekisteri/en/general/legislation/ Accessed Apr 15, 2016

21. Teppo L, Pukkala E and Lehtonen M: Data quality and quality control of a population-based cancer registry. Experience in Finland. Acta Oncol 33: 365-369, 1994.

22. Krieger JN, Egan KJ, Ross SO, Jacobs R and Berger RE: Chronic pelvic pains represent the most prominent urogenital symptoms of 'chronic prostatitis'. Urology 48: 715-722, 1996. 